\title{
Écrire et penser le genre en contextes postcoloniaux, sous la direction de Anne CASTAING et Élodie GADEN
}

\section{Emanuela Cacchioli}

\section{(2) OpenEdition}

\section{Journals}

\section{Edizione digitale}

URL: http://journals.openedition.org/studifrancesi/15897

DOI: 10.4000/studifrancesi. 15897

ISSN: 2421-5856

\section{Editore}

Rosenberg \& Sellier

\section{Edizione cartacea}

Data di pubblicazione: 1 décembre 2018

Paginazione: $535-536$

ISSN: 0039-2944

\section{Notizia bibliografica digitale}

Emanuela Cacchioli, «Écrire et penser le genre en contextes postcoloniaux, sous la direction de Anne CASTAING et Élodie GADEN», Studi Francesi [Online], 186 (LXII | III) | 2018, online dal 01 janvier 2019, consultato il 06 janvier 2021. URL: http://journals.openedition.org/studifrancesi/15897 ; DOI: https:// doi.org/10.4000/studifrancesi. 15897

Questo documento è stato generato automaticamente il 6 janvier 2021.

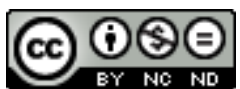

Studi Francesi è distribuita con Licenza Creative Commons Attribuzione - Non commerciale - Non opere derivate 4.0 Internazionale. 


\title{
Écrire et penser le genre en contextes postcoloniaux, sous la direction de Anne CASTAING et Élodie GADEN
}

\author{
Emanuela Cacchioli
}

\section{NOTIZIA}

Écrire et penser le genre en contextes postcoloniaux, sous la direction de Anne CASTAING et Élodie GADEN, Bruxelles, P.I.E Peter Lang, 2017, 326 pp.

1 È particolarmente interessante la collettanea curata da Anne CASTAING e Élodie GADEN in quanto affronta alcune riflessioni legate ai Gender Studies in relazione ai Subaltern Studies e ai Postcolonial Studies. Nello specifico, i due critici considerano le implicazioni femministe in un contesto non-occidentale. La prospettiva degli studi inseriti nel volume è duplice. Da un lato l'obiettivo è quello di cogliere le tensioni che emergono nella costruzione di un'alterità «genrée et racisée» (p.9), al fine di individuare le strategie di potere e i processi egemonici che caratterizzano in modo più o meno evidente la scrittura postcoloniale: i rapporti di dominio sono alla base di tutte le relazioni umane. Gli articoli non si propongono soltanto di identificare delle tendenze comuni, bensì di cogliere la peculiarità delle singole manifestazioni letterarie, dei processi creativi e, soprattutto, di indagare la specificità di ogni testo a partire dai valori, i saperi e la storia che hanno contribuito alla sua produzione. Il contesto culturale particolare permette di ripensare in modo originale le categorie uomo/donna, ma anche i rapporti di dominio che intercorrono tra di loro e l'influsso del colonialismo che agisce indirettamente. Per giungere a questa visione, occorre passare attraverso una serie di tappe che prevedono innanzitutto l'identificazione degli stereotipi, dei paradossi e degli «universalismes» (p. 11) del discorso coloniale. Occorre poi adottare un nuovo punto di vista: «se situer à partir $d u$ Sud» (p.11) per ripensare il genere, valorizzare l'eterogeneità delle identità e la rappresentazione letteraria. Analizzare le 
singole opere consente di trarre alcune riflessioni generali sulla resistenza della subalternità in un contesto extra-europeo e sulla sua rappresentazione in ambito letterario. Al fine di realizzare questa prospettiva "locale", gli autori ricorrono a differenti approcci che concorrono a comporre una visione originale e a valorizzarne l'ibridità: storico, antropologico, letterario, poetico, estetico, filosofico e cinematografico.

2 Il volume si suddivide in quattro sezioni. La prima dimensione considerata è quella storica. Hélène NICOLAS adotta uno sguardo antropologico in una prospettiva diacronica per spiegare i rapporti di dominio maschile su quello femminile che si manifestano in seno al popolo kanak della Nuova Caledonia. La spiegazione è da ricercarsi nella politica di riforma del matrimonio imposta dal colonizzatore. Anne CASTAING ci porta, invece, in India. La studiosa analizza le rappresentazioni linguistiche relative al femminile in un contesto di subalternità nella letteratura indiana dagli anni Venti a oggi. Per ripensare il genere, occorre considerare le scritture marginali perché sono le uniche in grado di offrire un'alternativa alla prospettiva parzialmente distorta che viene portata avanti dai testi "canonici". Il contributo di Tiziana LEUCCI si sofferma sul ruolo delle danzatrici dei templi e di corte, sempre all'interno della società indiana. Lo scopo dell'articolo è quello di tracciare un profilo di queste presenze femminili seducenti, ma colte e dedite all'arte, che sono state equiparate a prostitute ed emarginate per molti decenni. La loro figura è stata rivalutata a partire dagli anni Ottanta proprio per lo status privilegiato di letterate dotate di un minimo di autonomia.

3 La seconda parte ha come titolo «Métisser la nation» e contiene due contributi. Il primo, di Tina HARPIN, ci proietta nelle Antille, una terra da sempre associata al femminile. Si tratta di una dimensione complessa e ambivalente che presuppone sia una visione trionfalistica che un'immagine di sottomissione. Secondo la studiosa è necessario uscire da questa visione stereotipata per giungere infine a una riflessione originale e più veritiera sulla relazione tra uomo e donna. Emmanuelle RADAR si occupa al contrario di scrittrici franco-vietnamite, dalle cui opere emerge la voce maschile attraverso il procedimento della ripetizione. La relazione che si instaura tra il maschile e il femminile, tra il Vietnam e la Francia, tra il passato coloniale e il presente nelle periferie delle città europee tesse un filo che non sfocia in una rivendicazione, ma che consente di elaborare una forma di resistenza di fronte al potere coercitivo della collettività.

4 La terza sezione si concentra sulla poetica postcoloniale e sui diversi tipi di produzioni artistiche che caratterizzano la contemporaneità: il romanzo, la novella, il racconto, ma soprattutto la cinematografia. Xavier GARNIER si sofferma sui romanzi di Bessora, scrittrice originaria del Gabon, in cui l'umorismo e l'autoderisione offrono l'occasione per interrogare la società e le sue fratture. Nel contesto postcoloniale, contraddistinto dalle ineguaglianze sociali, la sensualità può diventare un gioco che garantisce la sopravvivenza. Per questo motivo, seduttore e sedotto fanno leva su genere e razza per uscire vincitori dalla battaglia. Christine LORRE-JOHNSTON analizza un romanzo dello scrittore canadese Highway in cui due fratelli subiscono violenze sessuali in un pensionato nel quale passano l'adolescenza, ma riescono a uscirne grazie al recupero del folklore. La studiosa mette in rilievo come il contesto coloniale sia caratterizzato dalla convivenza di opposti (rivolta e resistenza, ambiguità e compromesso) senza che questi sfocino in opposizione binaria. Sarah-Anaïs Crevier GOULET sceglie come focus due romanzi, un racconto e una novella di Marie NDiaye, autrice di origine senegalese 
che mantiene un certo distacco dall'Africa e che non ha un unico modello femminile di riferimento, bensì lascia spazio a una pluralità di donne determinate che resistono indipendentemente dal ruolo svolto (sorelle, sirene o streghe). Chiude la sezione il contributo di Mehdi DERFOUFI. Il critico esamina due film hollywoodiani, La Route des Indes e Zero Dark Thirty, usciti a trent'anni di distanza l'uno dall'altro. Nei due lungometraggi è messa in scena l'estetica della malinconia, che si rafforza con il passare del tempo fino a iscrivere il bianco nella condizione di oppressione.

5 La quarta parte esamina il genere nel contesto postcoloniale attraverso il «prisme des singularités culturelles» (p.18) per giungere infine a una decolonizzazione. Corinne FORTIER effettua un excursus storico e antropologico sull'uso del velo nelle diverse epoche, religioni e società. All'analisi della polisemia del termine nel Corano, si affianca una disamina della nozione di harem. Quest'ultimo aspetto scardina la distinzione tra pubblico e privato e riporta la discussione sull'opposizione tra uomo e donna. Il velo non è sempre un atto di sottomissione al dominio patriarcale, ma può divenire un gesto di resistenza attiva contro l'ordine coloniale e la ricerca di giustizia sociale. Segue un articolo a quattro mani di Hélène MARTIN e Patricia Roux che ripercorre le traduzioni francesi delle opere teoriche del femminismo postcoloniale, pubblicate recentemente. Di questi testi si indaga la correlazione con i rapporti di potere, la spinta a mantenere le specificità locali ed evitare generalizzazioni e il processo di costruzione del sapere. Rada IVECović si sofferma sul fatto che le figure femminili e le migrazioni sono in grado di scardinare i rapporti tra le istituzioni frutto di una costruzione storica. Queste due categorie partono da una posizione marginale di esclusione, ma in grado di fare comunità e divenire norma. Il volume si chiude con un'intervista a Maxime CERVULlE, Cornelia MÖSER e Karima RAMDANI sulla questione della traduzione di testi prodotti in contesto postcoloniale e su come si possano rendere in un'altra lingua nozioni e concetti portatori di una visione del mondo, dei rapporti sociali e di genere peculiari di un determinato ambito.

6 Si tratta di un volume particolarmente interessante sia per l'approccio plurale dei contributi sia per gli spunti di riflessione originali relativi a un tema inesauribile, ma anche per la relazione che intercorre tra gli studi postcoloniali e i Subaltern Studies. Una collaborazione tra settori del sapere che si rivela particolarmente feconda per rendere la complessità del reale. 